\title{
Vivencias de las prácticas sexuales asociadas al BDSM: los límites contemplados dentro de lo Sano, Seguro y Consensuado
}

\author{
Experiences of sexual practices associated with BDSM: the limits \\ contemplated within the bounds of what is Healthy, Safe and \\ Consensual
}

Victoria Ríos (https://orcid.org/0000-0002-5530-4058) ${ }^{1}$

Alejandrina Amundaray (https://orcid.org/0000-0002-9593-8899) ${ }^{1}$

Ysamary Arenas (https://orcid.org/0000-0002-3991-1368) ${ }^{1}$

\begin{abstract}
BDSM sexual practices related to sadomasochism, domination and submission, represent a vast range of possibilities in terms of paraphernalia, associated emotions, uses and applications that are as diverse as the human beings who practice it. Taking this into account, we conducted a study to understand the practices related to BDSM, under the perspective of its own practitioners. For this, a qualitative methodology was used, with an emergent and projected type of design. We covered 8 in-depth interviews on men and women from Venezuela and the data was analyzed through the constant comparison method in order to generate grounded theory, this paper comes up as part of a larger study. Among the most outstanding findings were the importance of limits, some as irrefutable an others that are meant to break, and the importance of the physical and mental health conditions in the practice. Key words Psychology, Sadomasochism, BDSM, Sexuality, Constructionism
\end{abstract}

Resumen Las prácticas sexuales BDSM relacionadas con el sadomasoquismo, la dominación y la sumisión, representan un abanico vasto de posibilidades en cuanto a parafernalia, emociones asociadas y usos tan diversos como los seres humanos que lo practican. Debido a esto, realizamos un estudio para entender las prácticas sexuales relacionadas al BDSM, bajo la mirada de sus propios practicantes. Para ello se empleó una metodología cualitativa con un tipo de diseño emergente $y$ proyectado. Se realizaron 8 entrevistas a profundidad a hombres y mujeres en Venezuela, se analizaron los datos a través del método de comparación constante con el fin de generar teoría fundamentada. Este artículo surge como parte de los resultados de un estudio mayor. Entre los hallazgos más resaltantes se encontró la importancia a los límites, algunos como irrefutables y otros que se intentan traspasar y lo primordial de la práctica en condiciones de salud física y mental.

Palabras clave Psicología, Sadomasoquismo, BDSM, Sexualidad, Construccionismo
${ }^{1}$ Departamento de Ciencias del Comportamiento. Universidad Metropolitana. Caracas Venezuela. victoriacrios@gmail.com 


\section{Introducción}

Las prácticas BDSM (Bondage, Dominación, Sumisión, Sadismo y Masoquismo) suelen ser comprendidas bajo dos paradigmas teóricos. Por un lado, desde la psicopatología y perversión basados en que el sadomasoquismo es una perversión y catalogado dentro del DSM V como un trastorno mental ${ }^{1}$ y por otro lado desde el construccionismo social como parte de la subcultura del BDSM, quienes apuntan a una mirada inclusiva e innovadora de la sexualidad, la cual se contempla como un arte que involucra nuevas formas de expresión y nuevas elecciones sexuales ${ }^{2}$. Adicionalmente, resulta llamativo que hemos encontrado investigaciones que sugieren la inexistencia de una relación entre la patología y la práctica $\mathrm{BDSM}^{3}$. Debido a esto surge la necesidad de realizar este trabajo, para comprender la perspectiva de sus protagonistas. Asimismo, encontramos un déficit importante de información $y$ estudios realizados en castellano y principalmente en Venezuela. Para realizar esto, se empleó una metodología cualitativa, con el objetivo de lograr teoría fundamentada. Los datos se recolectaron entre diciembre de 2015 y marzo de 2016, como parte de un estudio mayor denominado NO ES SEXO, ES UN ESTILO DE VIDA: PODER, DOLOR Y PLACER EN LA RELACIÓN SEXUAL. El presente artículo se basa en parte de los hallazgos expuestos en la tercera dimensión "Prácticas relacionadas al BDSM".

Más adelante se puede apreciar cómo, más allá de las posturas teóricas, las prácticas BDSM pueden ser vivenciadas como una subcultura; se trata de una comunidad que se rige bajo ciertas normas y costumbres; utilizan diversos tipos de parafernalias y métodos particulares para relacionarse sexualmente. Estas prácticas conllevan un proceso paso a paso que puede tener infinitas variaciones y posibilidades. A su vez, estas prácticas suponen la vivencia del dolor como algo placentero, entendido por cada practicante como algo totalmente subjetivo a pesar de la fisiología.

\section{Marco referencial}

Las manifestaciones de sadismo y masoquismo han estado presentes a lo largo de la historia de la humanidad. El sadomasoquismo como tal fue adquiriendo una connotación sexual, gracias a dos influyentes autores de obras literarias: Donatien Alphonse François de Sade a finales del siglo XVIII, debido a sus relatos en los que describe prácticas sexuales que incluyen violen- cia, humillación, parafilias y otro tipo de escenas extravagantes y por Leopold von Sacher-Masoch, escritor de novelas que narran prácticas de sumisión y masoquismo sexual, que datan de un siglo posterior a Sade. Más adelante, el sadomasoquismo se considera como una desviación sexual y se categoriza como un trastorno mental dentro de las parafilias en los manuales diagnósticos de patologías mentales, lo cual se mantiene hasta la fecha de hoy.

Por otra parte, como ya mencionamos anteriormente, existen planteamientos que conciben al sadomasoquismo como una subcultura y otorgan a estos individuos su propio discurso, alejándose así del enfoque de perversión o enfermedad. Foucault ${ }^{2}$, considera que la sexualidad se va desarrollando históricamente a partir de la necesidad de crear nuevas elecciones sexuales. Análogamente, Rubin, explica que la sexualidad no puede plantearse meramente en términos biológicos sino que se debe tomar una postura construccionista de la misma, enfocándose en la significación que construye el individuo a partir de su vivencia y el intercambio con la sociedad ${ }^{4}$.

El BDSM es un fenómeno relativamente nuevo, originario de Estados Unidos y Europa alrededor de los años 50 con el que muchos individuos pueden relacionarse sin importar su preferencia sexual $^{5}$. Esta subcultura abarca al sadomasoquismo, y se erige como la contraposición de la perspectiva patológica de este fenómeno. El BDSM abarca al sadomasoquismo, sin embargo; el BDSM es una dimensión mayor que contiene una multiplicidad de prácticas, de concepciones y de categorías. En primer lugar, denota la sumisión/dominación, el bondage, el dolor, la cultura del cuero, el intercambio de poder, los juegos de roles y los fetiches. Otra diferenciación común, es asociar el BDSM con el juego, por lo que los practicantes de esta sexualidad suelen referirse a "fiestas de juego" refiriéndose a lugares de encuentro para este fenómeno e incorporando el uso de juguetes, ropas y otro tipo de parafernalias. Por su parte, las prácticas pueden permutar desde las más comunes como el bondage o los látigos a las menos habituales como jugar con temas de incesto. Es importante destacar que para cualquier tipo de práctica del BDSM se debe seguir el mantra fundamental de la misma, siempre deben ser "seguras, sanas y consensuadas".

Ahora bien, el BDSM pareciera proponer entonces una nueva forma de elección de sexualidad, con una vasta variedad de posibilidades. Esto se acerca a lo propuesto por Michel Foucault cuando plantea que la sexualidad es un arte, una 
creación propia del ser humano que por consiguiente, puede abarcar una amplia gama de posibilidades ${ }^{2}$. Foucault concuerda en que el sadomasoquismo comprende estas nuevas posibilidades de ejercer el placer, ya que para él, lejos de ser una práctica agresiva mediante la cual los individuos descargan violencia, se refiere más bien a una producción creativa a través de la erotización del cuerpo. Para ello utilizan partes desconocidas de su cuerpo, prácticas no acostumbradas, objetos extraños, etc.

Finalmente, el abordaje de este fenómeno se realizó desde el Construccionismo social, un enfoque que hace especial énfasis en la comprensión de la experiencia social individual a través del lenguaje, para generar una teoría colectiva. Se trata de la generación de teoría para la reconstrucción social. De forma similar, esta investigación pretendió, precisamente, comprender las realidades de un conjunto de sujetos sadomasoquistas en relación con su medio y su experiencia subjetiva para generar una teoría que reúna los puntos en común, logrando de esta forma una reconstrucción social de la vivencia sadomasoquista. Bajo este punto de vista, lo que se toma en cuenta como "realidad", y se considera como una verdad primordial acerca de cómo funciona el hombre, es un subproducto de la construcción social $^{6}$.

El fundamento tomado en cuenta para decidir el construccionismo como abordaje paradigmático fue justamente la estigmatización y patologización a las que son expuestas las identidades y prácticas sexuales no convencionales en las sociedades occidentales. El positivismo implicaría la connotación de desviación, la condición de "fuera de la norma" que se traduciría en el sesgo de los datos. De esta forma, la invitación al lector es llevar el análisis y la discusión de los hallazgos presentados más allá de la dicotomía normalidad-patología y adentrarse en las vivencias y significados que estos sujetos expresan.

\section{Metodología}

Este estudio adoptó un enfoque de investigación cualitativa. El fenómeno del sadomasoquismo se ha abordado desde distintas perspectivas, para nosotras toma mayor envergadura la mirada de sus propios representantes y practicantes, ya que "la realidad que importa es lo que las personas perciben como importante"'. Nuestro interés se basó en producir datos descriptivos derivados de entrevistas a profundidad semiestructuradas con los participantes, de modo que intentamos realizar un análisis lo más parecido a la realidad posible. Aprovechando la virtualidad, escribimos a más de 100 personas en sus redes sociales abarcando FetLife (red social para personas con fetiches específicos), Facebook y Twitter. Con respecto a estos posibles participantes, de primera mano encontramos respuestas hostiles y suspicaces, de todos los que contactábamos pocos respondían y menor número accedía a los términos de la entrevista, esto principalmente porque alegaban que querían proteger su identidad. Sin embargo, hubo participantes que se mostraron más abiertos, sirviendo como porteros para conseguir a otros. Estos participantes también nos recomendaron bibliografía e incluso nos invitaron a un taller de bondage y shibari que se realizó en el Parque del Este (Caracas, Venezuela). Es importante acotar que asistimos, nos recibieron amablemente, nos presentaron a los demás practicantes y nos explicaron distintas técnicas de bondage. Esto nos permitió compartir con ellos en su contexto y lograr mayor profundidad en sus vivencias.

Utilizamos un muestreo de caso-tipo, dado que se buscó obtener información sobre las vivencias, experiencias y significados de sadomasoquistas o practicantes de BDSM. Asimismo, se empleó un muestreo en cadenas o en redes, ya que algunos participantes nos proporcionaron el contacto para acceder a otros y poder entrevistarlos. Se llevó a cabo un muestreo teórico con la intención de guiar el proceso de recolección de datos bajo la constante comparación con la teoría emergente. En este caso, la teoría generada a partir del análisis de cada entrevista determinó el tipo de características que buscaríamos en el siguiente entrevistado, como el sexo, el rol con el cual se identifican dentro del BDSM y la orientación sexual.

La muestra estuvo constituida por voluntarios mayores de edad (mayor de 18 años), venezolanos, independientemente del contexto en el que se encontraran viviendo actualmente, y que se identificaban a sí mismos como sadomasoquistas, practicantes del sadomasoquismo o practicantes de BDSM (Cuadro 1).

Por otra parte, nuestra investigación cuenta con un diseño emergente en el cual los datos se basaron principalmente en las anécdotas y experiencias de vida. En esta misma línea, debido a que el diseño permite que la investigación se vaya adaptando a los datos proporcionados, se replantearon los objetivos, cambiando el nombre de practicantes de sadomasoquismo por prac- 
Cuadro 1. Descripción de los participantes de la investigación.

\begin{tabular}{|l|l|l|l|l|l|}
\hline \multicolumn{1}{|c|}{$\mathbf{N}^{\circ}$} & \multicolumn{1}{c|}{ Nombre } & \multicolumn{1}{c|}{ Edad } & \multicolumn{1}{c|}{ Sexo } & \multicolumn{1}{c|}{ Rol } & \multicolumn{1}{c|}{ Orientación sexual } \\
\hline 1 & Anna & 20 & F & Sumisa/masoquista & Homosexual \\
\hline 2 & Octavio & 46 & M & Dominante & Heterosexual \\
\hline 3 & Alejandro & 20 & M & Switch & Bisexual \\
\hline 4 y 5 & Elisa y Pascal & 35 y 38 & F y M & Sumisa y Dominante & Heterosexual \\
\hline 6 & Petra & 23 & F & Sumisa & Heterosexual \\
\hline 7 & Katerina & 35 & F & Dominante & Bisexual \\
\hline 8 & Xavier & 33 & M & Sadista & Bisexual \\
\hline
\end{tabular}

Fuente: creación propia.

ticantes de BDSM ya que ellos se significan de esta manera y de esta forma se pudo mantener el estudio más fiel a dicha comunidad. Se utilizó además un diseño proyectado en donde establecimos como límite de tiempo un año. De esta manera, se trabajó en función de este tiempo para culminar el estudio, realizando un análisis y redacción de los resultados sin aspirar a completar una teoría ${ }^{8}$.

En relación al análisis de los datos, la información obtenida en cada entrevista fue analizada antes de continuar con la siguiente, respetando así el diseño emergente. El método utilizado fue el de comparaciones constantes llegó a un nivel avanzado de codificación axial. El núcleo central de donde se deslindaron las demás categorías fueron las vivencias relacionadas al BDSM y de estas surgieron cuatro grandes dimensiones: construcción de significados asociados al BDSM, "Está dentro de tu personalidad, dentro de tu identidad, forma parte de ti”, prácticas relacionadas al BDSM y BDSM dentro del contexto venezolano y extranjero. En este artículo se desarrollará solo la tercera dimensión.

Finalmente, llevamos a cabo diferentes acciones para asegurar la calidad y validez de esta investigación, entre estas: fue necesario que las investigadoras procesaran los datos, cada una por separado, de forma equivalente para luego someter los resultados a revisión; adicionalmente, se incorporó una auditoría externa que implicó la revisión del proceso completo por un experto para constatar que fue llevado de manera adecuada. Utilizamos la corroboración estructural, por medio de la cual se reúne la información emergente y se realizan constantes vinculaciones $\mathrm{y}$ conexiones, de forma tal que se fue conformando una estructura cuya base es la misma evidencia, y los datos se "soporten conceptualmente". Asimismo, realizamos el chequeo de los resul- tados del análisis y la teoría emergente con los mismos participantes, para asegurar que las interpretaciones hechas se aproximen lo suficiente a la comprensión de sus vivencias.

Por otra parte, procuramos una muestra variada, incluyendo a participantes de distintos sexos, orientación sexual y buscando abarcar toda la gama de roles que se emplean en las prácticas de BDSM, de modo que se tenga mayor posibilidad de que el estudio se aplique a otros contextos o muestras.

\section{Resultados}

El término BDSM es producto de una construcción social que abarca diversas maneras de relacionarse sexualmente asociadas con el sadomasoquismo, los juegos de roles y de intercambio de poder. Entre estas expresiones hallamos las prácticas sexuales BDSM que son tomadas por quienes se consideran partícipes con particular premeditación y precaución, es decir que de ninguna forma son llevadas a cabo a la ligera e implican un acuerdo previo o consenso entre los individuos involucrados.

\section{Condiciones para practicar BDSM}

Los participantes de este estudio enfatizaron que para llevar a cabo estas prácticas, se debe seguir una serie de pasos. En primer lugar, es fundamental que ambas personas se encuentren sanas, tanto mental como físicamente lo cual implica entre algunas cosas tener estabilidad emocional, no estar molesto, no estar deprimido, etc. En segundo lugar, la práctica debe realizarse de manera progresiva, es decir, se deben ir probando los límites y umbrales de dolor gradualmente y por último, debe existir un acuerdo que está rela- 
cionado intrínsecamente con el tema del consenso, en donde ambos participantes establecen sus gustos y límites.

Encontramos que la sanidad mental es considerada como primordial en el momento de la práctica, lo que ellos llaman "sesiones", pues está directamente relacionado con la posibilidad de establecer y mantener los límites, tal como lo expresa Elisa Si no estás bien psicológicamente, entonces, ¿cómo defines tú donde están tus limites sanamente? Si tú, eh, si tu raciocinio y tus parámetros están desfasados. Esta regla de oro pudiera explicar por qué en el estudio de Wismeijer los practicantes del BDSM resultaron ser personas mentalmente más equilibradas y se situaron como más confiados y serenos que los no practicantes ${ }^{10}$. Desde el punto de vista de los participantes, esto desmitifica la creencia de que las personas envueltas en ellas actúan de manera precipitada y descuidada o de que tienen problemas emocionales y trastornos psicológicos.

Similarmente, las sumisas y masoquistas lejos de la creencia común, deben tener alta autoestima debido a que deben poseer mayor nivel de resistencia y fortaleza mental para realizar este tipo de prácticas si yo voy a una sesión, y utilizo el acto de humillación a esa persona, ¿cómo lo va a tomar? Se va a destruir, se va a desmoronar. $Y$ voy a tener a una persona dañada en mis manos. $Y$ no puede. Al contrario, el sumiso tiene que tener una autoestima muy alta para ser sumiso (Katerina).

Asimismo, la sanidad física también es importante en la práctica. Una condición inherente a esto es la ausencia de consumo de drogas u otras sustancias que alteren el juicio de la realidad y la percepción, como plantea Pascal tú no puedes sesionar a alguien estando ebrio, ¿por qué? Porque tú pierdes la percepción, tú no te vas a dar cuenta de lo que estás causando esto hace referencia al principio fundamental del BDSM, que habla de prácticas sanas, seguras y consensuadas. Para estas personas realizar la práctica con el juicio alterado, quiere decir que se está poniendo en peligro a la otra persona o a sí mismo, debido al alto riesgo que estas prácticas conllevan, por lo que tiene sentido entonces que los practicantes tomen medidas de seguridad mucho más elaboradas que en una práctica sexual convencional.

Otro elemento a considerar en el ámbito físico es tomar en cuenta siempre el estado de la persona, es decir, lo que es satisfactorio un día no lo es siempre Xavier expresa que también depende del día puede ser que yo te diga, por lo menos... en su mayoría yo siempre he tenido sumisas que son mujeres, sabes, tu cuerpo sufre muchos procesos en un mes. Hay ciertos días donde aguantas más, ciertos días que aguantas menos, o sea, eso depende mucho de muchos factores pues

Según lo expresado por los participantes, cada quien tiene un funcionamiento fisiológico y psicológico particular, por lo que la práctica, la experiencia y la persona definen la manera en que se sesiona. De esta forma, otra condición para practicar BDSM es realizarlo de manera progresiva y paulatina involucrando diversos procesos en la forma en la que el amo y el sumiso se conocen, compartiendo acerca de sus preferencias y de sus límites para verificar la compatibilidad de ambos antes de establecer un contrato y llevar a cabo una sesión. Este proceso implica una especie de entrenamiento a través de aproximaciones sucesivas, sin llegar directamente a la conducta final esperada.

Dicho entrenamiento pudiese involucrar cuestionarios sobre intereses y experiencias previas que permiten una aproximación inicial al sumiso e incluso pueden usarse como métodos de descarte de personas con características patológicas: Yo primero hablo con ellos, por ejemplo, por chat, por donde me consiguen, mando este cuestionario que es un cuestionario de muchas preguntas donde hago descartes psicológicos viendo que tanto nivel pueden tener que prácticas han tenido y si es algún loco de carretera (Katerina).

Otro paso en el entrenamiento progresivo puede ser la doma La doma es un tiempo reglamentario [...] el dominante y el sumiso tienen un encuentro real que ya es para discutir el formulario. Si pasa ese momento, uno le manda por lo menos a hacer pruebas en su casa fotos y eso para ver si las hace y si es real todo, porque lo que vas haciendo son como pequeñas pruebas hasta llegar a una prueba real que es el momento de la primera sesión. Se entiende por doma un período de tiempo de prueba y de discusión de los gustos y límites del sumiso y dominante, hasta llegar a un punto de consenso o acuerdo sobre los mismos, lo cual quiere decir que el dominante ha aceptado al sumiso, acto que posteriormente se formalizará en público, para luego realizar el contrato.

Estos cuidados que se tienen antes de formalizar una relación de sumiso-dominante pudieran estar relacionados, entre otros factores, con algunos de los comentarios de los participantes que concuerdan en que estas prácticas pueden impactar a muchas personas, y precisamente para no impactar al nuevo practicante deben ser practicadas "poco a poco". Pascal hace referencia a esto un verdadero dominante que vaya a enseñar a alguien o tenga una sumisa, para él, debe ir pro- 
gresivamente. Yo no te puedo agarrar el primer día, "ah okey, vamos a sesionar" la agarro y agarro el látigo, no.

Parece ser que para muchos las prácticas BDSM son esencialmente estructuradas. En el inicio de una relación de dominación y sumisión se deben fijar previamente unas pautas y se deben realizar de forma progresiva, desde las más básicas y "vainilla" (término que para los participantes equivale a convencional) hasta las más complejas, tratándose de un proceso en el que se toma en cuenta múltiples factores. En muchos casos se trabaja el umbral de dolor, que denota el límite del sumiso-masoquista, con el fin de aumentarlo progresivamente, con respecto al umbral que tenía al inicio de la relación dominación-sumisión, hasta encontrar un nuevo límite.

\section{El contrato}

Otro de los principios del BDSM es el consentimiento. Sobre esto Petra comenta que $o b-$ viamente nada se va a hacer obligado, ninguna relación, nada de lo que se hace, se hace obligado y Octavio agrega Siendo sumisa acepta ser azotada en la espalda, azotada en las nalgas, bofeteada, o me dice, yo no acepto y no firmo ese contrato. No la puedes obligar a estar contigo. Esto nos da a entender que este contrato es un acuerdo conversado y premeditado, donde se pone sobre la mesa las experiencias previas, los gustos específicos y los límites de cada quien. Incluso, según algunos participantes puede ser similar al acuerdo que hacen las parejas convencionales antes de tener relaciones sexuales Un acuerdo, tal cual, como cuando en algún momento tú estás con alguna persona y estas saliendo y de repente deciden algún día tener relaciones pues, funciona tal cual igual, la diferencia es que la relación va a ser con ciertas actividades (Xavier).

Este acuerdo puede ser conversado antes de iniciar la práctica como tal entonces antes de llegar a una sesión uno debe conversar mucho con, debe haber mucha conversación entre ambos, para ver qué te gusta, qué no te gusta, qué me gusta a mí, qué no me gusta a mí (Pascal). Para la mayoría de los practicantes y según lo revisado en la teoría, el acuerdo siempre debe ser conversado, sin embargo; para dos de los entrevistados pueden no existir acuerdos previos sobre los límites, Ida refiere nunca hubo como una propuesta de... ite gustaría hacer esto? Tal cosa, sino como que ella tomó el control de todo, lo hizo todo ella y Alejandro plantea que cuando no se conversan, se van tanteando poco a poco $y$ bueno, eso, cuando gen- eralmente no tiene ese mutuo acuerdo, es como ir tanteando la zona. De repente... bueno, teniendo sexo muerdes a la persona y ves cómo reacciona... uno por lo menos va viendo qué cosas van gustando o... que no, y uno simplemente va hasta el punto en que... en que esa persona dice 'No, ya va'

Tomando esto en cuenta, nos planteamos la posibilidad de que los practicantes de BDSM sean más conscientes de lo que buscan y esperan, en relación a los no practicantes, pues suelen establecer mayor comunicación en sus relaciones, ya que deben expresar de antemano sus deseos, miedos y expectativas, lo cual pudiera traer como resultado el desarrollo de una mayor profundidad en el vínculo de estas parejas. Esto puede estar relacionado con lo expresado por Elisa son más intensos, tú ves a una pareja BDSM que lleva 6 meses y ves cómo interactúan y tu juras que tienen 5 años juntas...conozco parejas que tienen un nivel de interacción y de trato que es, eh, de parejas que tienen años...

\section{Los límites}

Al hablar de acuerdo y consentimiento, consideramos necesario mencionar los límites, que son definidos por los participantes como el punto máximo de dolor al que llega una persona o como toda práctica sexual que quede excluida de los gustos y preferencias contempladas en el acuerdo, cuando hablamos de limites hablamos en el sentido de cosas que tu no practicas, o sea, que jamás vas a practicar (Petra). Es imprescindible acotar que todos los participantes hacen especial énfasis en el tema de los límites, Elisa aclara $e l$ CSS, el sano, seguro y consensuado. Los límites son absoluta y totalmente básicos dentro del BDSM. Así, traspasar los límites sin premeditarlo significaría romper ese acuerdo y por ende violar la voluntad del otro, lo cual por supuesto genera displacer y malestar.

Hay quienes piensan que irrespetar los límites es equivalente a vivir el BDSM de manera insana si alguno de esos dos se rompe o la persona no tiene la confianza de que van a respetar los límites o de que van a respetar la palabra de seguridad, eso lo que te demuestra es que el dominante no es una persona... seria. No es una persona que vive esto, sino que es una persona que lo está viviendo de manera insana (Xavier).

Para estas personas, al hablar de límites, no solo estamos hablando de lo que se acuerda antes de llegar a sesionar, sino que también tomamos en consideración los límites que se pueden establecer durante una sesión o el trascurso del momento en 
que se llevan a cabo las prácticas BDSM. Debido a esto, los practicantes utilizan, como un método rápido y eficaz de comunicación durante la sesión, la palabra de seguridad. Para Castleman, es una señal de "detente" empleada por el sumiso que automáticamente implica que cualquier actividad que se esté realizando debe pararse ${ }^{11}$. Esta puede ser usada en cualquier momento durante la sesión y se suele elegir alguna palabra como "luz roja" en vez de una negativa como "detente, para o no". Petra argumenta Pero entonces tú tienes esa palabra de seguridad para si ya llegaste a tu punto límite que ya no aguantas más, que quieres parar o que algo no te gustó verdad, dices la palabra de seguridad y Xavier concuerda Uno de alguna manera ya tiene riendas sueltas porque sabes que está la palabra que si en algún momento te excedes, sucede eso y todo se detiene. No tienes que estar como 'ay, mira ¿esto está bien? ¿Esto está un poquito más, un poquito menos?'.

Según el discurso de los participantes existen dos elementos que pueden repercutir en la rigidez o flexibilidad con que se imponen los límites: el miedo y la confianza. Ante esto, Xavier comenta ¿Por qué tú te fijas un límite? Yo diría por miedo. O sea, todos nos fijamos ciertos limites por miedo... O sea, un ejemplo mira, yo no uso el metro después de las 9 de la noche... el metro está abierto hasta las 11, nadie te está poniendo ese limite, tú mismo te lo estás poniendo por una cuestión de que bueno, mira, miedo a que me pueda pasar algo...., lo que puede sugerir que una vez perdido el miedo pudiera haber mayor disposición y apertura a ir más allá de los límites.

En contraposición a esto aparece la confianza. Llevar una relación de este tipo no solo significa mantener una comunicación más abierta y profunda, sino que además implica altos niveles de confianza entre la sumisa y el dominante. Da la impresión de que mientras mayor sea la confianza entre las partes, mayor será la disposición y apertura de ambos para cruzar los límites y llegar más lejos en la práctica, tal como expresa Petra $l o$ que es el aceptar y el poder probar una gran cantidad de cosas, si es una persona por la cual a lo mejor no sientes afecto o no tanto, puede que no te importe tanto la otra persona o que tanto esté disfrutando, así mismo sea una persona con la cual quieras más o... incluso si es una persona con la cual de repente tienes mucha confianza uno logra llegar, por así decirlo, más lejos. Así, se considera que mientras más lejos llegue el límite de una sumisa-masoquista, mayor será su nivel de confianza con su dominante, dándole una connotación de mérito y reconocimiento.
Como se ha observado, el BDSM es una práctica compleja, por ello, los límites no se quedan atrás, no todos los límites son iguales, Elisa señala que existen limites suaves y existen limites duros. Los límites duros son los que jamás se van a realizar, están prohibidos estos pueden tratarse de la intervención en otros contextos de la vida del practicante -incluso para quienes lo vivencian como un estilo de vida- como la familia, el trabajo, la economía y los amigos jamás me va a poder ordenar en esas áreas como tal del no vas a llamar a tu mamá. Vas a llamarla dentro de 15 dias, porque no, porque eso es caca, no se toca... los amigos. Todas las relaciones amistosas con las cuales tengo años. Nada de eso puede tocar (Elisa). Por otro lado, los límites suaves se refieren a los que no descartas desde el principio, puede incomodar pero no son un problema, ante esto Octavio refiere que los puedes ir llevando poco a poco.

Ante esto, nos preguntamos por qué los límites no se dan de manera tajante y ya, lo prohibido que no se debe hacer, pero consideramos que el BDSM es una práctica que se trabaja de manera progresiva, que fomenta la experimentación de nuevas sensaciones, gustos y actividades que antes no se pensaban que se podían realizar y que generarían placer.

En esta misma línea, otros lo denominan como dos tipos de límites: los franqueables y los infranqueables. Los primeros, hacen referencia al proceso del sumiso en el cual va probando sus límites, en el cual puede cambiar lo que consideraba prohibido o displacentero para poder darse la oportunidad de probar con nuevas prácticas Yo creo que, un sumiso o una sumisa de alguna manera está en un descubrir, está en un experimentar... Está en un conocer Xavier también agrega que Entonces obviamente esto es un proceso de ambos, también el dominante conocer más a la sumisa y todo eso, indicándonos que tanto sumisos como dominantes poseen límites.

Nos llama la atención la manera en que los participantes plantean la cuestión de los límites: como algo que se debe respetar, pero que al mismo tiempo parte primordial de la práctica es poder traspasarlos. De hecho, el BDSM pareciera ofrecer un abanico de posibilidades ilimitadas, donde todo está permitido siempre y cuando sea sano, seguro y consensuado. Se encuentra incongruente el factor de que los límites sean considerados un elemento fundamental, adyacente y obligatorio en la práctica, pero que de alguna forma por debajo se busquen romper o transgredir, convirtiéndose el BDSM en una práctica del "todo se puede". 
Pareciera entonces que la motivación principal de estas personas para probar algo distinto a la práctica sexual convencional yace en la sensación de falta e insatisfacción que les ha dejado su experiencia sexual previa, lo cual los lleva a sentir la necesidad de emprender una búsqueda de aquello que les falta y que el BDSM les brinda, la relación sexual a partir de la constante búsqueda de lo que no está, que se encuentra más allá de los límites.

\section{Conclusiones}

La realización de esta investigación nos llevó a entender que para aproximarnos y comprender la vivencia de las personas practicantes del sadomasoquismo era necesario abarcar una categoría más amplia, llevándonos a reorientar nuestra atención hacia el BDSM; el cual tiene como base el intercambio de roles y poder. Es decir, que desde las etapas más tempranas de la realización de esta investigación, resultó primordial abordar el BDSM con una metodología que nos permitiera trabajar con la subjetividad de quienes la protagonizan y al mismo tiempo adaptar y amoldar el diseño de la investigación según los resultados que surgieran, dentro de un tiempo limitado, como lo hace el diseño emergente proyectado.

La técnica de la entrevista a profundidad fue esencial para captar el discurso de cada participante e intentar realizar el mayor acercamiento a sus vivencias. Más allá de las entrevistas a profundidad, la inmersión en el campo y las experiencias que tuvimos junto con los participantes nos ayudaron significativamente a comprender mucho más sobre la vivencia del BDSM de estas personas en un ámbito más cotidiano y natural, proporcionándonos una información primordial que no nos daban los resultados de las entrevistas. De esta forma, haber empleado la metodología cualitativa, bajo el método de teoría fundamentada nos permitió generar conocimientos acerca de las vivencias de los practicantes del BDSM y las prácticas relacionadas al mismo.

Se podría decir que, en este caso, lo que más sobresale es lo paradójico de la cuestión de los límites en la práctica BDSM, ya que el acuerdo y el establecimiento de los mismos son una regla fundamental incluso antes de iniciar las sesiones, más al mismo tiempo el buscar ir siempre más allá de los límites es igualmente una característica esencial de estas prácticas.

Ahora bien, desde el psicoanálisis, la explicación del BDSM como una perversión, remite a algo que se refleja en estas vivencias, la renegación de la ley. Se niegan los límites establecidos en la práctica sexual convencional, haciendo a un lado la prohibición y dando paso a la permisividad dentro del abanico de posibilidades en la práctica sexual BDSM, para luego crear ellos mismos sus propios límites. Todo está permitido bajo una premisa de sano, seguro y consensuado, como una forma de normativizar sus actividades. Mientras tanto, los planteamientos postmodernos se refieren al BDSM como un mundo de posibilidades, una cultura sexual creativa e innovadora, se encuentran infinitas formas alternativas de conseguir placer sexual, muchas veces apartando las prácticas sexuales convencionales, entre las cuales se encuentra el coito. Tal como expresa Ruiz-Tagle "en el BDSM el uso de prótesis ha liberado a las personas de las limitaciones de la anatomía, quitándole al pene su exclusividad"12.

Todo lo anterior podemos extrapolarlo a la situación moderna actual, en la cual "el cielo es el límite". Nos permite dar pie al más allá, al querer siempre lo que no está, quizá lo que para los participantes falta en la práctica sexual vainilla o convencional y buscan constantemente en el BDSM. Nos hemos volcado bajo un patrón de adicción a la comodidad y la seguridad, y el temor a sentir miedo, lo aceptamos como si fuera lógico, o al menos inevitable, hasta tal punto que contribuimos a normalizar el estado de emergencia ${ }^{13}$.Vivimos tan obsesionados por la seguridad que corremos el riesgo de perder nuestra libertad y para ello nos creamos nuestras propias reglas que nos permitan sentir un "sano, seguro y consensuado", normativizamos lo no permitido para que sea posible.

Asimismo, vivimos bajo sociedades líquidas, cambiantes, superfluas, en donde el vínculo se ve debilitado, en donde se da la posibilidad de ser el objeto sexual del otro, más allá del vínculo ${ }^{13}$, claro, porque queremos todo para ya, lo imposible para "ahorita". Bajo este discurso donde todo es posible, antes muchos deseos sexuales se quedaban en las fantasías ahora es posible actuarlos. Ahora todo se puede comprar y vender con tal de satisfacernos. ¿Son nuestros deseos tan distintos o la única diferencia es atreverse a vivirlos? ¿Qué tanto nos parecemos o diferenciamos al final del día? Esta pregunta resulta interesante a la luz de lo "cerrados" que podemos ser aún como individuos y como colectivos. Vivimos en un "vale todo" que segrega constantemente al diferente planteando lo que podríamos llamar una paradoja de la época, el discurso del todo es posible frente al no aceptar la diversidad. Nos preguntamos si la cuestión está en la posibilidad de nuevos 
discursos pero con muros tan altos que no permiten la inclusión ¿no se tolera la diferencia en el endogrupo, pero se respeta el derecho a crear uno nuevo? Nos atrevemos a pensar que precisamente este discurso postmoderno, bajo el cual todo es posible, es el que da cabida a la diversificación en la manifestación de la sexualidad del ser humano, permitiendo que surja el BDSM como subcultura, algo que en otras épocas históricas, posiblemente, no hubiera sucedido.

\section{Colaboraciones}

V Ríos y A Amundaray concibieron la idea, realizaron el análisis e interpretación de los datos y la redacción del artículo. Y Arenas Asesoró todo el desarrollo de la investigación y revisó y aprobó la versión que se publicará. Coordina la línea de investigación. 


\section{Referencias}

1. Moser C, Kleinplatz P. El DSM-IV y las parafilias: Un argumento para su retirada. Revista de Terapia Sexual y de Pareja 2004; 19:31-56.

2. Foucault M. Sex, power and the politics of identity. En: Rabinow P. Michel Foucault: Ethics, subjectivity and truth. Nueva York: The New Press; 1997. p. 163-173

3. Richters J, de Visser R, Rissel C, Grulich A, Smith A. Demographic and Psychosocial Features of Participants in Bondage and Discipline, "Sadomasochism" or Dominance and Submission (BDSM): Data from a National Survey. Journal of Sexual Medicine 2008; 5(7):1660-1668.

4. Rubin G. Thinking Sex: Notes for a Radical Theory of the Politics of Sexuality. In: Vance C. Pleasure and Danger: Exploring Female Sexuality. London: Pandora; 1984. p. 267-293.

5. Weiss M. Techniques of Pleasure: BDSM and the Circuits of Sexuality. Durham: Duke University Press; 2011.

6. Gergen K. Construccionismo social: aportes para el debate y la práctica. Bogotá: Universidad de los Andes; 2011.

7. Taylor S, Bodgan R. Introducción a los métodos cualitativos de información. 2a ed. Buenos Aires: Paidós; 1994.

8. Inche J, Ramírez D. El diseño de la investigación cualitativa. Planteamiento para la Investigación 2004; 1(1).

9. Hernández R, Fernández C, Baptista P. Metodología de la Investigación. $4^{\mathrm{a}}$ ed. México: Mc Graw Hill; 2006.

10. Wismeijer A, van Assen M. Psychological characteristics of BDSM practitioners. Journal of Sexual Medicine 2013; 10(8):1943-1952.

11. Psychology today (Internet). Nueva York: Sussex Publishers, LLC; 1967. A loving introduction to BDSM. [accesado 2012 Jun 15]. Disponible en: https://www. psychologytoday.com/blog/all-about-sex/201206/loving-introduction-bdsm

12. Ruiz-Tagle J. Creatividad y estereotipia en el sadomasoquismo. Nomadias 2013; (17):179-188.

13. Bauman Z. Miedo líquido. La sociedad contemporánea y sus temores. Barcelona: Paidós; 2007.

Artigo apresentado em 02/04/2018

Aprovado em 22/10/2018

Versão final apresentada em 19/02/2019 